\title{
Calidad del servicio educativo utilizando los estándares del SINEACE, el caso de la carrera administración de negocios internacionales de un ISTP
}

\author{
Educational Service Quality Using The Sineace Standards (National System of \\ Evaluation, Accreditation And Certification of Higher Education) - Case study: \\ International Business Management at a Higher Technological Institute
}

Verónica Díaz Noel ${ }^{1}$

\section{RESUMEN}

En la presente investigación, se ha evaluado la calidad del servicio educativo de la carrera Administración de Negocios Internacionales de un Instituto Superior Tecnológico Privado (ISTP); se trata de una investigación descriptiva, dicha evaluación se realizó tomando como base los estándares del SINEACE (Sistema Nacional de Evaluación, Acreditación y Certificación de la calidad Educativa), y se utilizaron diversas técnicas como encuestas, entrevistas, análisis documental, observación in situ.

La investigación concluye en que el servicio educativo de la carrera evaluada tiene un nivel de calidad bajo, y, en consecuencia, no se encuentra lista para obtener la acreditación.

Finalmente, se brindan algunas recomendaciones para elevar dicho nivel de calidad y así lograr la acreditación requerida.

\section{Palabras clave}

Calidad educativa, evaluación del servicio educativo, acreditación, SINEACE.

\section{ABSTRACT}

This descriptive research aims to assess the quality of educational service of the career of International Business Management provided at a Higher Technological Institute. This study was carried out on the basis of the SINEACE standards (National System of Evaluation, Accreditation and Certification of Higher Education); for this purpose, various techniques were used such as surveys, interviews, documentary analysis, in situ observation.

The research concludes that the educational service of the career under study has a low quality level; therefore, it is not ready to obtain the accreditation.

Finally, some recommendations are provided to increase such quality level and achieve the required accreditation.

\section{Keywords}

Educational quality, Evaluation of educational service, Accreditation, SINEACE (National System of Evaluation, Accreditation and Certification of Higher Education).

\footnotetext{
Magister en Administración de Empresas de Centrum - PUCP - Maastricht, egresada de la maestría en Educación con mención en Evaluación y Acreditación en la Calidad de la Educación de UNMSM, titulada en Ingeniería Electrónica de UNMSM, Directora de Carreras en el ISTP D. Alcides Carrión.
} 


\section{INTRODUCCIÓN}

La acreditación en la calidad de la educación es un tema que en muchos países ha cobrado fuerza en los últimos tiempos, razón por la cual se consideró relevante realizar la evaluación con fines de acreditación de la carrera Administración de Negocios Internacionales de un ISTP. La acreditación puede tener muchas ventajas tanto académicas como comerciales, pero cabe mencionar que no es útil si la institución solo crea documentos que no los aplica en la realidad, es decir, la institución debe primero decidir si su objetivo es solo comercial, o si por el contrario es asumir la calidad y la mejora continua como un estilo de vida. Cabe resaltar que el hecho de ir por el camino de la calidad tiene recompensas comerciales a largo plazo y sostenibles en el tiempo.

La comunidad educativa del ISTP percibe una mejora en la calidad del servicio educativo con respecto a hace 3 años, pero la institución aún no ha sido acreditada; además, teniendo en cuenta que la acreditación es un medio para implementar la cultura de calidad, se ha concluido en la necesidad de aprobar la evaluación con respecto a los estándares del SINEACE (antes CONEACES); para ello, el primer paso es determinar la situación actual a través de una autoevaluación, y el segundo paso es diseñar y ejecutar las medidas correctivas necesarias para obtener dicha acreditación.

Por otro lado, debido a la coyuntura que se vive actualmente en el Perú, en la que el gobierno y muchas instituciones educativas están trabajando en pro de la calidad educativa, se ha determinado implementar un sistema de mejora continua utilizando la herramienta Cuadro de Mando Integral e iniciando con la evaluación diagnóstica que se presenta en esta investigación.

\section{EL PROBLEMA}

\section{Problema General}

¿Cuál es el nivel de calidad del servicio educativo de la carrera Administración de
Negocios Internacionales del ISTP con respecto a los estándares del SINEACE (CONEACES) para la evaluación y acreditación en el año 2015?

\section{Objetivo General}

Identificar, especificar y precisar el nivel de calidad del servicio educativo de la carrera Administración de Negocios Internacionales del ISTP con respecto a los estándares del SINEACE (CONEACES) para la evaluación y acreditación en el año 2015.

\section{Justificación}

Considerando los beneficios de la Acreditación en la Calidad de la Educación, se fundamenta la necesidad de que la carrera Administración de Negocios Internacionales del ISTP sea evaluada utilizando los estándares del SINEACE, y de esta manera se puedan identificar las fortalezas y las oportunidades de mejora para que la carrera obtenga dicha acreditación, lo cual redundará en un incremento positivo de la imagen institucional en la percepción del público objetivo, y, en consecuencia, se tendrá un aumento en los resultados económicos para los accionistas. Por otro lado, la acreditación de la carrera Administración de Negocios Internacionales sentará las bases para la evaluación y acreditación del resto de carreras del ISTP.

\section{MARCO TEÓRICO}

\subsection{Antecedentes}

Dentro de los antecedentes consultados, podemos mencionar a Ospina (2011) quien desarrolla una investigación titulada Evaluación de la Calidad en Educación Superior, cuyo objetivo general fue identificar y determinar los criterios de evaluación de la calidad en educación; para ello, consideró como población a tres programas académicos de diferentes disciplinas pertenecientes a dos instituciones de educación superior, clasificados en cinco categorías: a) estudiantes, b) profesores, c) directivos y administrativos, d) egresados; y e) empleadores. De la población total, 677 personas, para los tres programas, 
se tomó por consenso de los respectivos comités de evaluación una muestra de 242 personas, equivalente al $36 \%$, conformándose finalmente los tres grupos académicos evaluadores según el ordenamiento por categorías; la metodología utilizada fue el estudio colectivo de casos, llegando a la conclusión de que existe una debilidad en indicadores para medir los factores de impacto, innovación, mejora permanente e internacionalización de la educación, por lo que se recomienda a los responsables de los lineamientos de acreditación fortalecer estos factores con el número suficiente de indicadores y con criterios comunes, válidos y confiables para la evaluación integral de la calidad de los programas.

Por otro lado, Vega (2010) realizó un estudio titulado La evaluación como estrategia de política educativa en el establecimiento de la calidad educativa en las universidades peruanas del estado, casos: Costa, Sierra, Selva, teniendo como objetivo general identificar y caracterizar la evaluación como estrategia de política educativa, en sus diversos aspectos, que permiten el establecimiento de la calidad educativa en las Universidades Peruanas del Estado. La población a la que se le aplicó el estudio fueron docentes de la costa, sierra y selva (20 docentes de cada región). El investigador concluyó que se debe capacitar a los miembros de la Comisión de Evaluación y Acreditación para que, siguiendo todos los pasos metodológicos, promuevan $y$ desarrollen políticas evaluativas en cada una de las unidades académicas y administrativas de las universidades y se pueda implementar y desarrollar una adecuada política de evaluación y acreditación debidamente contextualizada. Además, señala que se debe brindar una capacitación a todos los docentes y autoridades de las universidades para la formulación de políticas educativas y políticas evaluativas para proponer el desarrollo de cultura evaluativa de calidad, y así desarrollar a la institución universitaria en general y a las facultades en particular.

\subsection{Bases Legales}

- Ley N. ${ }^{\circ} 28044$, Ley General de Educación,

- Ley N. ${ }^{\circ} 28740$, artículo $18 .^{\circ}$,

- Decreto Supremo N. ${ }^{\circ}$ 018-2007-ED, artículo 41. .

\subsection{Bases Teóricas}

Águila (2005), en su ensayo El concepto calidad en la educación universitaria: Clave para el logro de la competitividad institucional, afirma que la calidad posee múltiples dimensiones, visiones $e$ interpretaciones. Para el autor, el problema no consiste en buscar una nueva definición de calidad, pues ya existen muchas en la literatura actual, sino en determinar aquella que más se ajuste a la realidad latinoamericana, sin olvidar que la calidad tiene que estar conjugada con la pertinencia y el impacto, pues no se puede concebir una institución de educación superior, de calidad que no sea pertinente en su entorno social.

Borroto \& Salas (2004), en el artículo Acreditación y Evaluación Universitarias, señalan:

la acreditación universitaria es el resultado de un proceso de evaluación y seguimiento metódico y voluntario de la institución de educación superior, que permite obtener información real y objetiva sobre la calidad de las instituciones y programas universitarios que desarrolla. La acreditación permite atestiguar ante la sociedad la calidad de los procesos que se llevan a cabo en dicha institución.

Según Trindade (1996), en su ensayo Evaluación institucional y calidad académica: resistencia y construcción, indica que el proceso de evaluación institucional debe estar orientado hacia un triple objetivo:

- Mejorar la calidad de la educación superior que desarrolla,

- Mejorar la gestión universitaria,

- Rendir cuentas a la sociedad, 
Tabla 1: DIMENSIONES, FACTORES, CRITERIOS Y ESTÁNDARES DEL SINEACE

\begin{tabular}{|c|c|c|c|}
\hline DIMENSIÓN & FACTOR & CRITERIO & $\begin{array}{c}\text { N’ }^{\circ} \text { DE } \\
\text { ESTÁNDARES }\end{array}$ \\
\hline \multirow{4}{*}{$\begin{array}{l}\text { GESTIÓN } \\
\text { INSTITUCIONAL }\end{array}$} & $\begin{array}{l}\text { 1.1. Proyecto } \\
\text { educativo } \\
\text { institucional }\end{array}$ & $\begin{array}{l}\text { El instituto elabora, aprueba y actualiza } \\
\text { permanentemente su misión, visión y valores, } \\
\text { en concordancia con las políticas nacionales y } \\
\text { propósitos institucionales, articulándolo con su } \\
\text { propuesta pedagógica y las demandas del entorno. }\end{array}$ & 3 \\
\hline & $\begin{array}{l}\text { 1.2. Organización } \\
\text { y gestión } \\
\text { administrativa }\end{array}$ & $\begin{array}{l}\text { Se cuenta con una estructura organizacional que } \\
\text { garantiza una gestión de calidad de los procesos } \\
\text { del IFD. }\end{array}$ & 5 \\
\hline & $\begin{array}{l}\text { 1.3. Gestión } \\
\text { docente }\end{array}$ & $\begin{array}{l}\text { El IFD dispone de políticas y procedimientos } \\
\text { para la selección, evaluación y promoción de } \\
\text { formadores. }\end{array}$ & 6 \\
\hline & $\begin{array}{l}\text { 1.4. Gestión } \\
\text { presupuestal }\end{array}$ & $\begin{array}{l}\text { Los recursoseconómicos del IFD son administrados } \\
\text { de manera eficiente. }\end{array}$ & 3 \\
\hline \multirow{6}{*}{$\begin{array}{l}\text { PROCEESOS } \\
\text { ACADÉMICOS }\end{array}$} & $\begin{array}{l}\text { 2.1. Diseño } \\
\text { curricular }\end{array}$ & $\begin{array}{l}\text { El instituto garantiza una formación integral y } \\
\text { actualizada, cumple con la implementación de } \\
\text { políticas curriculares y co-curriculares y adecúa } \\
\text { una metodología pertinente para su desarrollo. }\end{array}$ & 7 \\
\hline & 2.2. Admisión & $\begin{array}{l}\text { El IFD cuenta con una oferta educativa, selecciona } \\
\text { y admite ingresantes. }\end{array}$ & 2 \\
\hline & $\begin{array}{l}\text { 2.3. Enseñanza - } \\
\text { aprendizaje }\end{array}$ & $\begin{array}{l}\text { El IFD implementa, ejecuta, evalúa, actualiza y } \\
\text { mejora sus procesos académicos. }\end{array}$ & 7 \\
\hline & 2.4. Titulación & $\begin{array}{l}\text { La titulación refleja el éxito de la oferta educativa } \\
\text { institucional. }\end{array}$ & 2 \\
\hline & 2.5. Consejería & $\begin{array}{l}\text { El IFD apoya de manera personalizada, a aquellos } \\
\text { estudiantes que requieren asistencia en aspectos } \\
\text { académicos y otros, en aspectos que afectan su } \\
\text { rendimiento. }\end{array}$ & 2 \\
\hline & 2.6. Investigación & $\begin{array}{l}\text { El IFD ha definido políticas y estrategias para } \\
\text { promover el desarrollo de la investigación. }\end{array}$ & 5 \\
\hline \multirow{4}{*}{$\begin{array}{l}\text { SERVICIOS DE } \\
\text { APOYO }\end{array}$} & $\begin{array}{l}\text { 3.1. Desarrollo } \\
\text { del personal } \\
\text { administrativo }\end{array}$ & $\begin{array}{l}\text { El IFD dispone de políticas y procedimientos } \\
\text { para el desarrollo del personal administrativo, } \\
\text { facilitando el cumplimiento de los objetivos } \\
\text { académicos. }\end{array}$ & 3 \\
\hline & $\begin{array}{l}\text { 3.2. Sistema de } \\
\text { información }\end{array}$ & $\begin{array}{l}\text { El IFD garantiza una adecuada organización de su } \\
\text { sistema de información. }\end{array}$ & 4 \\
\hline & 3.3. Bienestar & $\begin{array}{l}\text { El IFD promueve la participación de los estudiantes } \\
\text { en actividades cocurriculares y los asiste en sus } \\
\text { necesidades para un buen rendimiento académico. }\end{array}$ & 4 \\
\hline & $\begin{array}{l}\text { 3.4. Infraestructura, } \\
\text { equipamiento } \\
\text { y tecnología }\end{array}$ & $\begin{array}{l}\text { El IFD tiene una infraestructura, equipamiento } \\
\text { y tecnología moderna para el desarrollo de sus } \\
\text { actividades académicas y administrativas. Realiza } \\
\text { labores de mantenimiento preventivo y correctivo } \\
\text { y aplica acciones de protección del ambiente. }\end{array}$ & 8 \\
\hline \multirow{3}{*}{$\begin{array}{l}\text { RESULTADOS E } \\
\text { IMPACTO }\end{array}$} & $\begin{array}{l}\text { 4.1. Imagen } \\
\text { institucional }\end{array}$ & $\begin{array}{l}\text { El IFD tiene una imagen posicionada dentro del } \\
\text { medio social, cultural y productivo de su localidad. } \\
\text { Establece su participación en la sociedad y busca } \\
\text { ejercer influencia positiva sobre su entorno. }\end{array}$ & 2 \\
\hline & $\begin{array}{l}\text { 4.2. Proyección } \\
\text { social }\end{array}$ & $\begin{array}{l}\text { El IFD expresa su responsabilidad social, a través } \\
\text { de programas de proyección en beneficio de la } \\
\text { comunidad. }\end{array}$ & 3 \\
\hline & 4.3. Egresados & $\begin{array}{l}\text { El IFD realiza el seguimiento de sus egresados, } \\
\text { los convoca y organiza a fin de tener información } \\
\text { sobre su experiencia laboral, actualización y } \\
\text { servicio de empleo. }\end{array}$ & 4 \\
\hline \multicolumn{3}{|c|}{$\begin{array}{ll} & \text { TOTAL } \\
\end{array}$} & 70 \\
\hline
\end{tabular}

Fuente: CONEACES (ahora SINEACE) 2014. 
En el ámbito mundial, son los Ministerios de Educación de los países los encargados de establecer los lineamientos para que las instituciones educativas obtengan la acreditación. En el Perú, esta función la cumple el SINEACE (Sistema Nacional de Evaluación, Acreditación y Certificación de la Calidad Educativa), el cual establece los criterios, estándares y procesos de evaluación, acreditación y certificación que aseguren los niveles de calidad que deben brindar dichas instituciones educativas.

El modelo de acreditación del SINEACE evalúa la calidad educativa a través de cuatro dimensiones:
a. Gestión institucional,
b. Procesos académicos,
c. Servicios de apoyo para la información profesional,
d. Resultados e impacto social.

Según el marco normativo del SINEACE, la acreditación es un proceso voluntario; pero la creciente necesidad por demostrar que una Institución Educativa Superior (IES) carrera o programa cumplen con los requisitos correspondientes al estatus que aspira hace de este proceso una exigencia para promover y asegurar la calidad de la educación de nuestras instituciones.

En la tabla 1, se presentan los factores de cada dimensión, los criterios que se consideran en cada factor y el número de estándares que integran cada factor.

\section{MATERIALES Y MÉTODOS}

En la investigación, se utilizaron diversos materiales y métodos, los cuales han permitido medir algunos indicadores de la variable Calidad del Servicio Educativo en más de una forma, ello ha enriquecido la investigación, ya que se ha podido triangular los resultados.

\section{MÉTODOS}

- Encuestas,

- Entrevistas,
- Análisis documentario,

- Observación.

\section{MATERIALES}

- Cuestionario de encuestas dirigidas a:
- Alumnos,
- Docentes,
- Personal Administrativo,
- Egresados,

- Cuestionario de entrevistas dirigidas a:
- Alumnos,
- Directivos,
- Personal administrativo,
- Padres de familia,
- Empleadores,

- Rúbrica de Análisis documentario,

- Lista de cotejo,

- Ficha de observación.

\section{RESULTADOS}

En las tablas 2, 3, 4 y 5 , se muestran los resultados para cada una de las dimensiones que señala el SINEACE, resultados que fueron obtenidos utilizando diversos instrumentos (cuestionarios de entrevistas, de encuestas, fichas de observación, listas de cotejo y rúbricas de análisis documental). Cabe mencionar que el utilizar más de un instrumento para medir cada dimensión, ha permitido llegar a conclusiones más confiables, ya que se han triangulado dichos resultados, es decir, se observa que con los diversos instrumentos los resultados son los mismos en más del $50 \%$ de las veces. En cada tabla solo se presenta los valores que han obtenido mayor porcentaje o el mayor puntaje según sea el caso.

Previo a la presentación de los resultados, es importante señalar que las dimensiones de la variable "Calidad del Servicio Educativo" pueden tomar los siguientes valores: Alto, Medio, Bajo y Muy Bajo. 
Tabla 2: Resultados de la dimensión "Gestión Institucional"

\begin{tabular}{|c|c|c|c|}
\hline Encuesta a Alumnos & $\begin{array}{c}\text { Encuesta a } \\
\text { Docentes }\end{array}$ & $\begin{array}{c}\text { Encuesta al Personal } \\
\text { Administrativo }\end{array}$ & Entrevista a Alumnos \\
\hline $41 \%$ & $46 \%$ & $44 \%$ & $55 \%$ \\
\hline Bajo & Bajo & Bajo & Bajo \\
\hline $12 \%$ & $25 \%$ & $11 \%$ & $25 \%$ \\
\hline Muy Bajo & Muy Bajo & Muy Bajo & Muy Bajo \\
\hline $\begin{array}{c}\text { Entrevista al Personal } \\
\text { Administrativo }\end{array}$ & $\begin{array}{c}\text { Entrevista al } \\
\text { Personal Directivo }\end{array}$ & Lista de Cotejo & $\begin{array}{c}\text { Rúbrica de Análisis } \\
\text { Documental }\end{array}$ \\
\hline $40 \%$ & $60 \%$ & 114 & 29 \\
\hline Bajo & Alto & Bajo & Medio \\
\hline $20 \%$ & $0 \%$ & & \\
\hline Muy Bajo & Bajo & &
\end{tabular}

Tabla 3: Resultados de la dimensión "Procesos Académicos"

\begin{tabular}{|c|c|c|c|}
\hline Encuesta a Alumnos & Encuesta a Egresados & Entrevista a Alumnos & Lista de Cotejo \\
\hline $47 \%$ & $47 \%$ & $75 \%$ & 116 \\
\hline Bajo & Bajo & Bajo & Bajo \\
\hline $6 \%$ & $10 \%$ & & \\
\hline Muy Bajo & Muy Bajo & & \\
\cline { 1 - 3 } & & &
\end{tabular}

\begin{tabular}{|c|c|}
\hline $\begin{array}{c}\text { Rúbrica de Análisis } \\
\text { Documental }\end{array}$ & Ficha de Observación \\
\hline 25 & 6 \\
\hline Muy Bajo & Bajo \\
\hline
\end{tabular}


Tabla 4: Resultados de la dimensión "Servicios de Apoyo"

\begin{tabular}{|c|c|c|c|}
\hline Encuesta a Alumnos & $\begin{array}{c}\text { Encuesta a } \\
\text { Docentes }\end{array}$ & $\begin{array}{c}\text { Encuesta al Personal } \\
\text { Administrativo }\end{array}$ & $\begin{array}{c}\text { Entrevista al Personal } \\
\text { Administrativo }\end{array}$ \\
\hline $36 \%$ & $59 \%$ & $40 \%$ & $100 \%$ \\
\hline Bajo & Bajo & Bajo & Muy bajo \\
\hline $7 \%$ & $18 \%$ & $20 \%$ & \\
\hline Muy Bajo & Muy Bajo & Muy Bajo & Ficha de Observación \\
\hline $\begin{array}{c}\text { Entrevista al Personal } \\
\text { Directivo }\end{array}$ & Lista de Cotejo & $\begin{array}{c}\text { Rúbrica de Análisis } \\
\text { Documental }\end{array}$ & 23 \\
\hline $100 \%$ & 138 & 18 & Medio \\
\hline Muy Bajo & Bajo & Medio & \\
\hline
\end{tabular}

Tabla 5: Resultados de la dimensión "Resultados e Impacto"

\begin{tabular}{|c|c|c|c|}
\hline $\begin{array}{c}\text { Encuesta a } \\
\text { Alumnos }\end{array}$ & Encuesta a Egresados & $\begin{array}{c}\text { Entrevista a Padres de } \\
\text { Familia }\end{array}$ & $\begin{array}{c}\text { Entrevista a } \\
\text { Empleadores }\end{array}$ \\
\hline $42 \%$ & $40 \%$ & $\mathbf{5 0 \%}$ & $\mathbf{5 7 \%}$ \\
\hline Neutro & Medio & Medio & Medio \\
\hline
\end{tabular}

\begin{tabular}{|c|c|c|}
\hline Lista de Cotejo & $\begin{array}{c}\text { Rúbrica de Análisis } \\
\text { Documental }\end{array}$ & Ficha de Observación \\
\hline 27 & 5 & 6 \\
\hline Muy bajo & Bajo & Bajo \\
\hline
\end{tabular}




\section{DISCUSIÓN}

Para la dimensión “Gestión Institucional”, se ha obtenido que tanto en la encuesta a los alumnos, la encuesta a los docentes como la entrevista a los alumnos y la entrevista al personal administrativo, los valores "Bajo" y "Muy Bajo" superan el 50\%, es decir, con 04 de 05 instrumentos se verifica que el nivel de calidad es "Bajo", ello sumado a que también con el instrumento "Lista de Cotejo" se obtuvo el valor "Bajo", cabe mencionar que con el instrumento "Rúbrica de Análisis Documental" se obtuvo el valor "Medio", ello se debe a que la carrera sí cuenta con algunos de los documentos que solicita el SINEACE para esta dimensión, pero tal como lo evidencian los resultados en las encuestas y entrevistas, la existencia de estos documentos no es percibida por los sujetos encuestados y entrevistados.

Lo expuesto anteriormente es reforzado por Ramirez \& Baidez (2011), quienes señalan que toda organización educativa debe implantar su "filosofía de acción" como marco de referencia de sus diversas acciones en función del logro de sus objetivos. A dicha filosofía de acción se le llamará la política general, de la cual surgen otras políticas estructurales y funcionales.

Con respecto a la dimensión "Procesos Académicos", se ha evidenciado tanto en la encuesta a los alumnos como en la encuesta a los docentes un resultado por encima del $50 \%$ entre los valores "Bajo" y "Muy Bajo", además, en la entrevista a los alumnos se obtuvo el $75 \%$ con el valor "Negativo" el cual es comparable o equivalente al valor "Bajo", a ello le añadimos el hecho de que con los instrumentos "Lista de Cotejo" y "Ficha de Observación" se obtuvieron los valores "Bajo" en ambos casos, $y$, por último, con el instrumento "Rúbrica de Análisis Documental" se obtuvo el valor "Muy Bajo", es decir que, con los 06 instrumentos aplicados, los resultados obtenidos fueron entre "Bajo" y "Muy Bajo", esto es comprensible porque a pesar de que se han hecho algunos esfuerzos por actualizar la malla curricular y capacitar a los docentes, aún falta desarrollar algunos aspectos que exige el SINEACE como la motivación a los docentes, el sistema de tutoría a los estudiantes, el soporte para que los docentes realicen investigaciones y el apoyo para que los egresados logren la titulación.

En ese sentido, Toro (2012) ratifica que en los procesos académicos deben estar incluidos cuatro tipos de procesos, algunas veces relacionados:

- Procesos requeridos para la realización de los programas académicos formales que la institución ofrece (diseño curricular, proceso de admisión, proceso de enseñanza - aprendizaje).

- Procesos de apoyo y bienestar a los estudiantes (tutoría).

- Procesos asociados a la investigación (investigación).

- Procesos asociados a otros servicios de tipo educativo o profesional que la IES ofrece a la comunidad, como la educación continua o consultoría especializada.

Con relación a la dimensión "Servicios de Apoyo", si bien es cierto que la dimensión ha obtenido el $43 \%$ entre los valores "Bajo" y "Muy Bajo", es decir, por debajo del 50\%, encontramos que "Medio" es el siguiente valor con mayor porcentaje, es decir, la mayoría de los alumnos no perciben una mala calidad en esta dimensión, pero tampoco la perciben con una alta calidad, esto se debe a que aunque por un lado se han realizado mejoras significativas en la infraestructura y en el mobiliario del ISTP, por otro lado, es contrarrestado con servicios negativos como, por ejemplo, la mala atención que se tiene en la caja. Por otro lado, tanto en la encuesta a docentes como en la encuesta al personal administrativo, los valores "Bajo" y Muy Bajo" superan el 50\%, a ello se añade el hecho de que en las entrevistas al personal directivo y administrativo se obtuvo como resultado el valor "Muy Bajo" con un $100 \%$, esto se debe básicamente a que la institución no cuenta con un sistema de intranet tal como lo solicitan los estándares de evaluación. Además, con el instrumento "Lista de cotejo" el valor obtenido fue "Bajo", es decir, en 05 de los 07 instrumentos aplicados se obtuvo entre los valores "Bajo" y "Muy Bajo", en los otros 02 instrumentos se obtuvo el valor "Medio", esto se debe a que la institución cuenta con algunos 
de los documentos que esta dimensión exige, ello se verifica en el instrumento "Rúbrica de análisis documental", así como también cuenta con algunos de los servicios que esta dimensión solicita, como los servicios de salud, biblioteca, agua y luz, todo esto se evidencia en el instrumento "Ficha de observación".

Finalmente, para la dimensión "Resultados e Impacto" se ha verificado tanto en las encuestas a los alumnos, a los egresados, como en las entrevistas a los padres de familia y empleadores que se obtuvo como resultado el valor "Medio" o "Neutro", es decir, la percepción de los encuestados y entrevistados no es ni de una baja ni alta calidad en esta dimensión; sin embargo, los instrumentos "Lista de cotejo", "Rúbrica de análisis documental" y "Ficha de observación" dieron como resultado los valores "Bajo" y "Muy Bajo", esto se debe a que, si bien es cierto que la percepción de los sujetos encuestados y entrevistados no es mala, la institución no cumple con ciertos aspectos que pide el SINEACE para esta dimensión como, por ejemplo, el sistema de seguimiento a los egresados y las actividades de proyección social.

\section{CONCLUSIONES}

Después de haber aplicado las encuestas, haber realizado algunas entrevistas, haber verificado la existencia de algunos documentos, haber analizado los documentos, y haber observado la existencia de ciertos espacios físicos llegamos a las siguientes conclusiones.

- El nivel de calidad del servicio educativo de la carrera Administración de Negocios Internacionales del ISTP es "Bajo".

- La carrera evaluada no se encuentra lista para obtener la acreditación según los estándares del SINEACE.

- La dimensión Gestión Institucional de la carrera Administración de Negocios Internacionales del ISTP tiene un nivel de calidad "Bajo".

- La dimensión Procesos Académicos de la carrera Administración de Negocios
Internacionales del ISTP tiene un nivel de calidad "Bajo".

- La dimensión Servicios de Apoyo de la carrera Administración de Negocios Internacionales del ISTP tiene un nivel de calidad "Bajo".

- La dimensión Resultados e Impacto de la carrera Administración de Negocios Internacionales del ISTP tiene un nivel de calidad "Bajo".

- La calidad del servicio educativo de la carrera Administración de Negocios Internacionales del ISTP es una variable que está compuesta de varias aristas, entre ellas se encuentra la satisfacción de los alumnos, docentes y egresados, los recursos físicos, materiales y humanos que la institución proporciona para brindar el servicio en mención y la imagen que la institución proyecta a la sociedad.

- La variable "calidad del servicio educativo" de la carrera Administración de Negocios Internacionales del ISTP requiere de un conjunto de indicadores para ser medida, es decir, la opinión de los stakeholders (alumnos, docentes, personal administrativo, directivos, padres de familia y empleadores), el cumplimiento de la documentación requerida y la comprobación de que la información que figura en dicha documentación es real y fidedigna.

\section{RECOMENDACIONES}

A fin de que la institución educativa asegure calidad en el servicio que brinda, y así poder obtener el sello de Acreditación, se presentan las siguientes recomendaciones:

- Para elevar el nivel de calidad de la dimensión Gestión Institucional, se recomienda:

- Elaborar el PEI en conjunto con los stakeholders, es decir uno o dos representantes de cada grupo. 
- Elaborar el PEI basándose en documentos como el Proyecto Educativo Nacional, investigaciones de mercado, y otros documentos que se consideren pertinentes. Archivar toda esta documentación, para posteriormente evidenciar su uso.

- Difundir los puntos más importantes del PEI a través de boletines, correos electrónicos, reuniones periódicas y otras acciones que la institución considere conveniente.

- Implementar el área de calidad, para que se encargue de supervisar en toda la institución el cumplimiento de lo requerido en los estándares del SINEACE.

- Para el caso de la dimensión Procesos Académicos, se recomienda:

- Implementar un plan de acompañamiento al docente, con el fin de asegurar el cumplimiento de lo programado en los sílabos, así como la aplicación de las estrategias metodológicas que están enfocadas hacia el logro de los aprendizajes.

- Implementar y ejecutar un programa de tutoría para todos los estudiantes, en sesiones individuales o grupales según sus necesidades.
- Brindar el soporte necesario a los docentes para que realicen investigaciones, y que luego estas puedan ser indexadas en páginas web reconocidas para este fin.

- Con respecto a la dimensión Servicios de Apoyo, se recomienda lo siguiente:

- Desarrollar y ejecutar un plan de capacitación y evaluación al personal administrativo.

- Implementar la intranet, y que esta sea funcional y transparente para todos.

- Brindar servicios de psicología dentro de los servicios de salud.

- Capacitar en temas de atención al cliente al personal de las áreas de Atención al alumno y Caja.

- Por último, para la dimensión Resultados e Impacto, se recomienda lo siguiente:

- Generar alianzas estratégicas con instituciones nacionales reconocidas por su prestigio, y hacer públicas estas alianzas a modo de reportajes.

- Implementar y ejecutar el planeamiento de las actividades de proyección social.

- Desarrollar e implementar el proceso de seguimiento al egresado. 


\section{REFERENCIAS}

Águila Cabrera (2005) El concepto calidad en la educación universitaria: Clave para el logro de la competitividad institucional. Documento elaborado para la revista Ibero Americana de Educación. OEI. http://www.rieoei.org/deloslectores/880Aguila.PDF

Borroto Cruz \& Salas Perea, Acreditación y evaluación universitarias. http://bvs.sld.cu/revistas/ ems/vol18_3 04/ems01304.htm

MINEDU: $\quad$ www.minedu.gob.pe/DeInteres/xtras/download.php?link=coneaces estandares presentacion\%20(4).pdf. Consultado el 12 de agosto del 2014.

Ospina R. (2011) Evaluación de la calidad en educación superior (tesis para obtener el grado de Doctor). Universidad Complutense de Madrid, España.

SINEACE: https://www.sineace.gob.pe/acreditacion/institutos-y-escuelas-de-educacion-superior. Consultado el 07 de enero del 2016.

Toro R. (2012) Gestión interna de la calidad en las instituciones de educación superior. Chile: RIL Editores.

Trindade H. (1996) Evaluación institucional y calidad académica: resistencia y construcción. En: Conferencia Regional sobre Políticas y Estrategias para la Transformación de la Educación Superior en América Latina y el Caribe. La Habana: CRESALC/MES.

Vega P. (2010) La evaluación como estrategia de política educativa en el establecimiento de la calidad educativa en las universidades peruanas del estado, casos: Costa, Sierra, Selva.

Fecha de recepción: 29-03-16

Fecha de aceptación: 18-04-16 